\title{
On the BIC for determining the number of control points in B-spline surface approximation in case of correlated observations
}

DOI: https://doi.org/10.1515/jogs-2020-0110

Received November 8, 2019; accepted February 10, 2020

Abstract: B-spline curves are a linear combination of control points (CP) and B-spline basis functions. They satisfy the strong convex hull property and have a fine and local shape control as changing one $\mathrm{CP}$ affects the curve locally, whereas the total number of CP has a more general effect on the control polygon of the spline. Information criteria (IC), such as Akaike IC (AIC) and Bayesian IC (BIC), provide a way to determine an optimal number of $\mathrm{CP}$ so that the B-spline approximation fits optimally in a leastsquares (LS) sense with scattered and noisy observations. These criteria are based on the log-likelihood of the models and assume often that the error term is independent and identically distributed. This assumption is strong and accounts neither for heteroscedasticity nor for correlations. Thus, such effects have to be considered to avoid underor overfitting of the observations in the LS adjustment, i.e. bad approximation or noise approximation, respectively. In this contribution, we introduce generalized versions of the BIC derived using the concept of quasi- likelihood estimator (QLE). Our own extensions of the generalized BIC criteria account (i) explicitly for model misspecifications and complexity (ii) and additionally for the correlations of the residuals. To that aim, the correlation model of the residuals is assumed to correspond to a first order autoregressive process $\mathrm{AR}(1)$. We apply our general derivations to the specific case of B-spline approximations of curves and surfaces, and couple the information given by the different IC together. Consecutively, a didactical yet simple procedure to interpret the results given by the IC is provided in order to identify an optimal number of parameters to estimate in case of correlated observations. A concrete case study using observations from a bridge scanned with a Terrestrial Laser Scanner (TLS) highlights the proposed procedure.

Gaël Kermarrec: Geodetic Institute, Leibniz University Hannover, E-mail: kermarrec@gih.uni-hannover.de

Hamza Alkhatib: Geodetic Institute, Leibniz University Hannover, E-mail: alkhatib@gih.uni-hannover.de
Keywords: BIC, B-splines approximation, Terrestrial Laser Scanning, Control Points, correlation, Variance Inflation Factor, AR(1), Information Criteria, BIC, Generalized BIC, quasi-likelihood

\section{Introduction}

The fitting of curves or surfaces through a set of noisy observations with splines appears in many disciplines from mathematics and engineering up to medical imaging. In engineering geodesy specifically, the polynomial B-spline basis functions have gained increased interest as the obtained B-spline curves or surfaces have an interesting fine shape control. Point clouds (PC) from TLS can be typically approximated with parametric regression B-splines, as proposed, for example, in Koch (2009). Two of the major applications are the modelling of 3D surfaces and the monitoring of deformations (Xu et al. 2018, Zhao et al. 2018).

Using the regression approach, a cost function, defined as the distance between the mathematical B-spline curve or surface and the noisy observations, is to be minimized. The Euclidian distance is often used (see, e.g., (Aigner and Jüttler 2009)). An ordinary least-squares (OLS) adjustment is performed (Koch 1999), where the parameters to be estimated are the so-called control points (CP), which define a rough sketch of the curve or surface. The goodness of the approximation is strongly impacted when a suboptimal total number of $\mathrm{CP}$ is chosen to weight the $\mathrm{B}$-splines basis functions. Unfortunately, focusing on the specific case of B-spline fitting of TLS measurements, this number is often chosen heuristically. In the worst case, this leads to a loss of information and, potentially, an inaccurate determination of deformations, as shown in (Zhao et al. 2018).

This major challenge can be solved within the context of model selection by using penalized model selection criteria. A selection based on such criteria is, thus, a choice of the best model according to some underlying definition of what optimality should be. Many penalized criteria have been proposed in the literature that start from 
different theoretical assumptions. They are based on either Bayesian statistics or the Kullback-Leibler divergence principles (KLD; (Kullback and Leibler 1951)), and lead in their simple form to the BIC (Schwarz 1978) and AIC (Akaike 1973), (Akaike 1974) respectively, see also Burnham and Anderson (2002). The AIC has been extended in many ways to strengthen the penalty term for model complexity and account for small sample size (Bozdogan 1987) or kurtosis (Yanagihara 2006). Criteria related to BIC include adjusted BIC (Sclove 1987), whose penalty term is lighter than BIC. Alternatives were proposed, using crossvalidation techniques (Dziak et al. 2018), risk inflation criterion (Foster and George 1994) or statistical learning approaches (Cherkassky 2007), which were applied to Bsplines fitting by Harmening and Neuner (2016) and Harmening and Neuner (2016) (2017). Such strategies remain computationally demanding and are often asymptotically equivalent to AIC or BIC (Dziak et al. 2018). Thus, the two basic information criteria (IC) are widely preferred, for the sake of simplicity.

In its usual formulation, the BIC does not consider heteroscedasticity (HC) or correlations of the error term (Burnham and Anderson 2002). Unfortunately, focusing on applications to determine the optimal number of $\mathrm{CP}$ to estimate for approximating TLS observations with Bspline curves or surfaces, this strong assumption of independently and identically distributed (i.i.d.) errors does not hold: range and angle measurements have different variances (Wujanz et al. 2017, Wujanz et al. 2018), and ranges were empirically shown to be correlated in Kauker and Schwieger (2017). Stochastic misspecifications coming from the observations, as well as to some extent from functional misspecifications, are translated into the residuals of the adjustment, which are used to estimate the BIC numerically. Moreover, even if a generalized LS estimator is used, the residuals remain always correlated (Koch 1999). To face this challenge in the specific context of regression, Lv and Liu (2014) investigated generalized expressions of the information criteria using the quasimaximum likelihood estimator (QMLE) (McCullagh 1983, Wedderbrun 1974). The use of QLE allows one to derive a specific bias correction, which takes into consideration model misspecifications by means of the $\mathrm{HC}$ of the residuals, still not accounting for the correlations.

Neglecting correlations in the generalized BIC formulation can lead to an overfitting of the observations when used to determine the number of CP for B-spline approximation. Indeed, correlations have a similar effect as reducing the available information of the observations (Holst and Kuhlmann 2016, Kermarrec and Schön 2016). As fitting the correlated noise is unfavourable to obtain smooth approximations and can additionally lead to detecting inexistent deformations for TLS applications, this should be avoided. Thus, in this contribution, we extend mathematically the formulation of the generalized BIC to account in a simple and numerically usable way for $\mathrm{HC}$ and correlations. To that aim, a correction of the generalized BIC based on an innovative proposal using the concept of variance inflation will be explained. This proposal grandly simplifies the computational burden linked to fully populated variance covariance matrices (VCM) associated with correlations (Kermarrec and Schön 2016, Kermarrec and Schön 2017). The extension of the criterion can be used within the context of regression or other model selection problematics. In this contribution, we will explicitly focus on the determination of the optimal number of $\mathrm{CP}$ for B-splines surface or curve approximations of TLS observations. The problematic of accounting for correlations and $\mathrm{HC}$ is a highly relevant research topic as they impact strongly the results of statistical tests for detection of deformations. In this contribution, and focusing on the determination of the optimal number of CP for TLS applications, we consider the number of observations as high enough, so that we will restrict ourselves to the study of BIC. Similar extensions as those proposed in this contribution can be easily derived for the AIC.

This contribution should not be considered, however, as a definition of "another" IC and an empirical proof of its superiority wrt. the usual BIC. Much more, we wish to provide keys to understand the differences between the different criteria, particularly in case of correlations. Thus, we intentionally adopt a didactical approach to provide useful guidance for model selection using the BIC and its generalized versions. We do not aim neither (i) to give a rule of thumb to fix the number of $\mathrm{CP}$, nor (ii) to claim about the superiority of generalized criteria in all cases. We provide orientations on how to interpret the results of different criteria in order to make an enlightened choice, as none of them is supposed to lead to the unknown truth (Gigerenzer and Marewski 2005), which is a nearly philosophical problem in estimating parameters.

The remainder of the contribution is as follows: in Section 2, we will introduce the BIC. The quasi-likelihood criteria will be introduced as a generalization for non-i.i.d. (independent and identically distributed) observations. In Section 3, the theoretical derivations will be applied to the specific case of determining the number of $\mathrm{CP}$ in the regression B-splines approximation. We will use simulations of true curves to explain the results obtained for the different criteria didactically. Section 4 is dedicated to a real case study of surface fitting using gridded TLS observa- 
tions from a bridge under load, which will conclude this contribution.

\section{Information Criteria: the BIC}

In this section, we propose to review the mathematical concepts behind the well-known information criterion BIC. The derivation of the generalized criteria to account for heteroscedasticity and correlations will be explained, introducing the model misspecification and complexity terms.

\subsection{The BIC: introduction}

From an observed sample of data $D$ of size $n$, we consider $k_{\text {total }}$ possible models $M_{k}, k=1 \ldots k_{\text {total }}$, each of them having a likelihood function $p_{r}\left(D \mid \mathbf{x}_{k} ; M_{k}\right)$ specified by the parameter vector $\mathbf{x}_{k}$ of length $p_{k}$. The log-likelihood function of one model is defined as $l\left(\mathbf{x}_{k}\right)=\log \left(p_{r}\left(D \mid \mathbf{x}_{k} ; M_{k}\right)\right)$. We call $\hat{\mathbf{x}}_{k}$ the maximum likelihood estimate (MLE) of $\mathbf{x}_{k}$. The Bayesian criterion of Schwarz (1978)(Burnham and Anderson 2002) is to choose a model for which

$$
B I C_{k}=-2 l\left(\hat{\mathbf{x}}_{k}\right)+\log (n) p_{k}
$$

is minimum, i.e. the estimated information loss is thus minimized. $B I C_{k}$ is composed of the log-likelihood and a penalty term $\log (n) p_{k}$, which depends on the size of the sample of data $D$ and the unknown number of parameters to estimate. Thus, the criterion is of the family of the "penalized log-likelihood criteria", as the AIC (Akaike 1973, 1974).

We will call the model, which minimized Equation (1) the "result of the model selection" in the following. Furthermore, $B I C_{k}$ will be simply called BIC for the sake of readability.

\subsection{Shortcomings of the BIC}

\section{The true model}

The general formulation of the BIC is $-2 \log \left(\lambda_{k, n}\right)$, where $\lambda_{k, n}$ is the posterior probability of the $k^{\text {th }}$ model (Atkinson 1980). The BIC is a Bayesian criterion (Burnham and Anderson 2002), which compares probabilities that each of the models under consideration is the true model that generated the data observed. It assumes inherently that the true model exists. Transposed to real data, this statement is clearly idealistic.

\section{Normality assumption}

$\lambda_{k, n}$ can only be approximated, using, e.g., Laplace's method, for which normality has to be assumed. Thus, this criterion is more accurate for large samples when the posterior density becomes dominated by the information from the data. Burnham and Anderson (2002) argued that this asymptotic strategy may penalize strongly large models in model selection and lead to the well-known underfitting of the BIC for large samples. However, BIC will always remain consistent: if the data-generating process is among the models under consideration, then with data size growing large, the data-generating model is selected by the BIC criterion.

\section{Model misspecifications}

Model misspecifications can be either related to an incorrect specification of:

1. the functional model: an inappropriate mathematical description of the relationship between observations and parameters, and/or

2. the stochastic properties of the error term. Exemplarily, the errors are assumed to be i.i.d. with a zero mean and a constant variance for BIC. This assumption is strong and may omit not only heteroscedasticity but also correlations, may they be mathematical, physical or coming from the approximation itself. If the penalty term is not accordingly corrected, all models derived with BIC will be, to some extent, misspecified.

A possibility to circumvent the shortcomings of the BIC is to derivate the criterion within a more general probability distribution by means of the quasi-likelihood criteria. This criterion will be called a GBIC for "generalized BIC".

\subsection{Quasi-likelihood estimator}

\subsubsection{Introduction}

The QLE method is a standard and popular method to estimate the parameters in a generalized linear model as no cost functions has to be determined and optimized (Wedderbrun 1974, Xu et al. 2013). The only assumption of the QLE approach is the relation between the variance and the mean of the observations, the simplest relationship being, for example, a proportionality.

In the following, we define a model of parameter adjustment as $\mathbf{y}=\mathbf{f}(\mathbf{x})+\varepsilon$, with $\mathbf{y}$ being the data $D$, also called vector of observations of size $n, \mathbf{f}(\mathbf{x})$ a vector of linear or nonlinear functions of $\mathbf{x}$-the $\mathrm{k}$-dimensional vector to be estimated-and $\varepsilon$ the random errors corresponding to $\mathbf{y}$. When the relationship between parameters and obser- 
vations is linear, we have $\mathbf{f}(\mathbf{x})=\mathbf{D x}$, with $\mathbf{D}$ being the socalled design matrix of size $\left(n \times p_{k}\right)$. In that case, Tibshirani and Knight (1999) defined the quasi-likelihood function $Q L F(\mathbf{x})$ as

$$
\frac{\partial Q L F(\mathbf{x})}{\partial \mathbf{x}}=\mathbf{D}^{\mathbf{T}} \Sigma_{\mathbf{y}}^{-\mathbf{1}}(\mathbf{x})(\mathbf{y}-\mathbf{D} \mathbf{x})
$$

with $\Sigma_{\mathbf{y}}(\mathbf{x})$ the diagonal variance matrix of $\mathbf{y}$. The quasimaximum likelihood estimator $\hat{\mathbf{x}}_{Q M L E}$ is obtained by letting the derivatives of $Q L F(\mathbf{x})$ with respect to $\mathbf{x}$ be set to 0 , i.e. $\left.\frac{\partial Q L F(\mathbf{x})}{\partial \mathbf{x}}\right|_{\mathbf{x}=\hat{\mathbf{x}}_{O M L E}}=\mathbf{0} . \hat{\mathbf{x}}_{Q M L E}$ is asymptotically unbiased and normally distributed in case of large $n$.

To compute $\hat{\mathbf{x}}_{Q M L E}$, the true but unknown distribution of the observations has to be approximated by a wide family of distribution, which may or may not include the true one. This family is often chosen to be the regular exponential family, whose density function of one measurement $y$ is given by:

$$
f(y \mid \theta)=\mathrm{e}^{y \theta-\mathrm{g}(\theta)}
$$

where $\theta$ is to be estimated and $g$ is defined so that $E(y)=$ $\mu=g^{\prime}(\theta), \sigma_{y}^{2}=\frac{d \mu}{d \theta}=g^{\prime \prime}(\theta)$, (Xu et al. 2013). Taking the Lebesgue measure, the Gaussian univariate density with a mean $\mu$ and variance $\sigma^{2}$ is a particular case of exponential family.

It can be shown that the QMLE is identical to the maximum log-likelihood estimation if, and only if, the family of distribution chosen for the independent observations is the one parameter exponential family, for example, a normal distribution with a known mean. In that case, the quasi maximum likelihood estimator $\hat{\mathbf{x}}_{Q M L E}$ becomes the solution of the normal equation of a linear LS adjustment.

\subsubsection{The generalized BIC}

For the presentation of the generalized BIC, we chose intentionally the same notation as Lv and Liu (2014) so that interested readers can easily refer to this publication for more information and the corresponding theorems. We start with $\mathbf{D}$, the previously defined design matrix, which entries mathematically link linearly the $n$ observations with the $p_{k}$ parameters to estimate. Following Lv and Liu (2014), the asymptotic covariance matrix of $\hat{\mathbf{x}}_{Q M L E}$ is $\Sigma_{Q M L E}=\mathbf{A}_{n}^{-1} \mathbf{B}_{n} \mathbf{A}_{n}^{-1}$.

$\mathbf{A}_{n}$ is the Hessian matrix of the Fisher information matrix and can be estimated by means of the VCM of $\mathbf{D}^{T} \mathbf{y}=$ $\mathbf{D}^{T} \mathbf{D x}$, where we assume that the observations $\mathbf{y}$ are following the exponential distribution, i.e. $\mathbf{A}_{n}=\mathbf{D}^{T} \sum(\mathbf{D x}) \mathbf{D}$ (see (Lv and Liu 2014), Equation (3) for the definition of $\Sigma(\mathbf{D x}))$. On the other hand, $\mathbf{B}_{n}=\mathbf{D}^{T} \Sigma_{\mathbf{y}} \mathbf{D}$ is the outer prod- uct of the Fisher information matrix. $\Sigma_{\mathbf{y}}$ is here the VCM of the observations.

A generalized BIC can be derived by introducing the $\log$-likelihood difference between $\hat{\mathbf{x}}_{Q M E}$ and $\hat{\mathbf{x}}_{Q L E}$, i.e. the deviation of the quasi-likelihood from its maximum. Using asymptotic expansion, Lv and Liu (2014) shows that, similarly to the BIC, a GBIC can be defined as:

$$
G B I C=-2 l\left(\hat{\mathbf{x}}_{Q L E}\right)+p_{k} \log (n)-\log \left(\left|\hat{\mathbf{H}}_{n}\right|\right)
$$

where $\hat{\mathbf{H}}_{n}$ are estimates of the so-called true covariance "contrast matrix" $\mathbf{H}_{n}$ defined as:

$$
\mathbf{H}_{n}=\mathbf{A}_{n}^{-1} \mathbf{B}_{n} \text {, i.e. } \hat{\mathbf{H}}_{n}=\hat{\mathbf{A}}_{n}^{-1} \hat{\mathbf{B}}_{n}
$$

and $|\bullet|$ is the determinant of the matrix. On the contrary to Equation (1), GBIC takes explicitly into account a model misspecification term by means of $\log \left(\left|\hat{\mathbf{H}}_{n}\right|\right)$. In case of a correctly specified model, $\hat{\mathbf{H}}_{n}$ is the identity matrix and GBIC simplifies to BIC.

Further extension of the GBICto account for model complexity

A further extension of the $G B I C$ refines the penalty term for model complexity to prefer models with less variability by assuming a prior assignment depending on the KLD between consecutive models. Using asymptotic expansion, an extension of the GBIC is given by:

$$
G B I C_{K L D}=-2 l\left(\hat{\mathbf{x}}_{Q L E}\right)+p \log (n)+\operatorname{tr}\left(\hat{\mathbf{H}}_{n}\right)-\log \left(\left|\hat{\mathbf{H}}_{n}\right|\right)
$$

This improved formulation has the following advantages:

1. it can be explicitly decomposed as a sum of a term for the goodness of fit (the log-likelihood), two terms for model complexity (the standard penalty term and the trace term) and a term for model misspecification (the log determinant term).

2. it is asymptotically equivalent to BIC for a correctly specified model.

\subsubsection{Estimation of the contrast matrix}

The generalized BIC criteria both involves the estimation $\hat{\mathbf{H}}_{n}$ of $\mathbf{H}_{n}$ in Equations (5) and (6) to obtain numerically the $\log$ of its determinant and its trace. In the following, we present a way to estimate both $\mathbf{A}_{n}$ and $\mathbf{B}_{n}$.

- Since $\hat{\mathbf{x}}_{Q M L E}$ is a consistent estimator of the true parameter vector $\mathbf{x}$, an estimate of $\mathbf{A}_{n}$ is given by $\hat{\mathbf{A}}_{n}=\mathbf{D}^{T} \Sigma\left(\mathbf{D} \hat{\mathbf{x}}_{Q M L E}\right) \mathbf{D} \mathrm{Lv}$ and Liu (2014). For LS adjustment and one model $k$, we have 
$\Sigma\left(\mathbf{D} \hat{\mathbf{x}}_{Q M L E}\right)=\frac{\left(\left(\mathbf{y}-\mathbf{D} \hat{\mathbf{x}}_{\mathbf{Q M L E}}\right)^{\mathbf{T}}\left(\mathbf{y}-\mathbf{D} \hat{\mathbf{x}}_{\mathbf{Q M L E}}\right)\right)}{n-p_{k}}$. This approximation corresponds to $\hat{\sigma}^{2}$, the a posteriori variance factor of the estimates for the ordinary LS estimator.

- One possibility to compute an estimator $\hat{\mathbf{B}}_{n}$ of $\mathbf{D}^{T} \Sigma_{\mathbf{y}} \mathbf{D}$ is to place the $\mathrm{i}^{\text {th }}$ squared error into the $\mathrm{i}^{\text {th }}$ row of the diagonal of the VCM using the ordinary LS residuals to estimate the errors, i.e.

$$
\Sigma_{\mathbf{y} \_H C}=\operatorname{diag}\left(\left(\mathbf{y}-\mathbf{D} \hat{\mathbf{x}}_{\mathbf{Q M L E}}\right) o\left(\mathbf{y}-\mathbf{D} \hat{\mathbf{x}}_{\mathbf{Q M L E}}\right)\right)
$$

where $o$ is the component-wise product (Ziwot and Wang 2003).

Equation (7) leads to a diagonal VCM and accounts for the heteroscedasticity of the residuals but not for correlations. As aforementioned, this simplification is in most cases unrealistic so that a computational feasible alternative has to be found to account for the lost of information induced by correlations.

\subsubsection{Taking correlations into account}

In the case of a large correlated sample dataset, the direct estimation of the $\operatorname{VCM} \Sigma_{\mathbf{y}}$ using the residuals $\left(\mathbf{y}-\mathbf{D} \hat{\mathbf{x}}_{Q M L E}\right)$ will yield large matrices, thus, leading to inaccuracies and computational difficulties. Fortunately, $\Sigma_{\mathbf{y}}$ is only involved in the computation of $\hat{\mathbf{B}}_{n}$ by means of $\mathbf{D}^{T} \Sigma_{\mathbf{y}} \mathbf{D}$. Consecutively, we propose to use the results of Kermarrec and Schön (2016), and place us in the case where the values contained in the columns of $\mathbf{D}$ are not randomly distributed but are either constant, linearly, quadratically or cubically increasing (or decreasing) sorted. Indeed, in such cases, it is possible to condense the information contained in $\Sigma_{\mathbf{y}}^{-1}$ into a diagonal matrix called $\Sigma_{\mathbf{y} \_ \text {equi }}^{-1}$, where the sum of the values of one line of $\Sigma_{\mathbf{y}}^{-1}$ corresponds to the diagonal value of the equivalent matrix $\Sigma_{\mathbf{y} \_ \text {equi }}^{-1}$, see Kermarrec and Schön (2016) and the reference inside for more details. This method is, thus, a powerful tool to approximate $\Sigma_{\mathbf{y}}$ simply by $\Sigma_{\mathbf{y} \_ \text {equi }}=\left(\Sigma_{\mathbf{y} \_ \text {equi }}^{-1}\right)^{-1}$, which is an easy to use diagonal VCM.

Using a parametric covariance family, the parameters of $\Sigma_{\mathbf{y}}$-and thus of $\Sigma_{\mathbf{y}}^{-1}$ - can be estimated from the observations. In this contribution, we propose to approximate the correlation structure of the residuals with an easy-to-use and understandable tool, the autoregressive model of the first order AR(1). This proposal, which assumes a weak stationarity (Box and Jenkins 1976), models the value of the residuals at time $t$ as a linear function of the value at $(t-1)$. The algebraic expression of the model is $x_{t}=\kappa+\rho x_{t-1}+\varepsilon_{t}$, where $\kappa, \rho$ are two coefficients to be determined by adequate methods and $\varepsilon_{t}$ is an i.i.d. error term independent of the value of the time series at time t, $x_{t} . \rho$ is called the autocorrelation correlation coefficient. The correlation matrix of the corresponding process has a Toeplitz structure which is given in Rao and Toutenburg (1999) by:

$$
\mathbf{Q}_{A R(1)}=\left[\begin{array}{cccccc}
1 & \rho & \rho^{2} & \cdots & \rho^{n-1} & \rho^{n} \\
\rho & 1 & \rho & \ddots & \rho^{n-2} & \rho^{n-1} \\
\rho^{2} & \rho & 1 & \ddots & \rho^{n-3} & \rho^{n-2} \\
\vdots & \ddots & \ddots & \ddots & \ddots & \ddots \\
\rho^{n-1} & \ddots & \ddots & \ddots & 1 & \rho \\
\rho^{n} & \rho^{n-1} & \ddots & \cdots & \rho & 1
\end{array}\right]
$$

The main advantage of the AR(1) over other correlation models is that an explicit inverse $\mathbf{Q}_{A R(1)}^{-1}$ exists:

$\mathbf{Q}_{A R(1)}^{-1}=\frac{1}{1-\rho^{2}}\left[\begin{array}{cccccc}1 & -\rho & 0 & \cdots & 0 & 0 \\ -\rho & 1+\rho^{2} & -\rho & \ddots & 0 & 0 \\ 0 & -\rho & 1+\rho^{2} & \ddots & 0 & 0 \\ \vdots & \ddots & \ddots & \ddots & \ddots & 0 \\ 0 & 0 & 0 & \ddots & 1+\rho^{2} & -\rho \\ 0 & 0 & 0 & \cdots & -\rho & 1\end{array}\right]$

Consecutively, once the parameter $\rho$ is estimated, the diagonal equivalent matrix is given by:

$$
\begin{gathered}
\mathbf{Q}_{A R(1) \_ \text {equi }}^{-1}=\frac{1}{1-\rho^{2}} \\
{\left[\begin{array}{cccccc}
1-\rho & 0 & 0 & \cdots & 0 & 0 \\
0 & (1-\rho)^{2} & 0 & \ddots & 0 & 0 \\
0 & 0 & (1-\rho)^{2} & \ddots & 0 & 0 \\
\vdots & \ddots & \ddots & \ddots & \ddots & 0 \\
0 & 0 & 0 & \ddots & (1-\rho)^{2} & 0 \\
0 & 0 & 0 & \cdots & 0 & 1-\rho
\end{array}\right]}
\end{gathered}
$$

Thus, assuming that for large matrices, the first and last terms of the diagonal that have different values than the rest can be neglected, we approximate $\mathbf{Q}_{A R(1) \_e q u}$ by $\mathbf{Q}_{A R(1) \_ \text {equi }}=V F \mathbf{I}$, where $\mathbf{I}$ is the identity matrix and the variance inflation factor is defined as $V F=\frac{1+|\rho|}{1-|\rho|}$. The corresponding VCM $\Sigma_{\mathbf{y} \_H A C}$ is obtained from Equation (7) by:

$$
\Sigma_{\mathbf{y}_{-} H A C}=V F \Sigma_{\mathbf{y}_{-} H C}
$$

where HAC is the abbreviation for "heteroscedasticity and correlation". Clearly, the formulation of Equation (8) has advantages over using fully populated VCM, particularly when estimating the log determinant of the contrast matrix. In the following, we call the corresponding criteria 
using this approximation $G B I C_{H A C}, G B I C_{K L D, H A C}$ (Equations (4) and (6)).

We propose to summarize the correlation structure of the whole residuals with an AR(1) process, which we justify for the sake of computational simplification. The authors are aware that this model remains an approximation of the true model. It is based on an extensive empirical fitting of the autocorrelation function for the case study under consideration in this contribution. This simplification is based on a parametric approach of the VCM by means of an AR(1) process, under the condition that the design matrix is not randomly filled. Other approaches could use, e.g., the Matérn covariance family (Kermarrec and Schön 2017) or maximum likelihood analysis.

When correlations are neglected, the aposteriori variance factor involved in the computation of $\hat{\mathbf{A}}_{n}$ is overestimated (Kermarrec and Schön 2017). Thus, the $V F$ in Equation (8) acts as correcting the terms $\operatorname{tr}\left(\hat{\mathbf{H}}_{n}\right)$ and $\log \left(\left|\hat{\mathbf{H}}_{n}\right|\right)$ of Equation (6) accordingly. This is similar to accounting "in an hidden way" for correlations (Kermarrec and Schön 2016) without having to introduce fully populated matrices. With this methodology, we are, thus, NOT neglecting correlations.

\section{Computational consideration}

When $n$ is large, the number of eigenvalues of $\hat{\mathbf{H}}_{n}$ close to 0 may be non-negligible so that the log determinant will tend to $-\infty$. The problem can be circumvented by computing the sum-of-log rather than the log-of-product, i.e. $\log (\Pi)=\sum \log$. Indeed, the determinant of a triangular matrix equals the product of its diagonal elements, i.e. the matrix's log-determinant is equal to the sum of their logarithm values. To that aim a lower-upper (LU) factorization can be used, while for positive definite matrices, a Cholesky factorization is typically more efficient.

Another possibility is to ignore the small eigenvalues of $\hat{\mathbf{H}}_{n}$ from a given threshold, which is sometimes empirically fixed based on the maximum eigenvalue of $\hat{\mathbf{H}}_{n}$. In this contribution, the LU factorization is employed to compute the $\log$ of the determinant.

\subsubsection{Interpretation of the generalized BIC}

Because of the improved penalty terms, the "best" model given by the usual BIC may not agree with the one of the generalized criteria. In case of agreement, however, it can be claimed that the optimal model is found, see Section 2.3.2. and (Lv and Liu 2014). The difference between BIC and the generalized criteria will depend on the respective strength of the model penalty and misspecification terms wrt. to the loglikelihood of the model.

1. If $G B I C_{H C} \neq G B I C_{H A C}$, the residuals can be considered as correlated enough to affect the determination of the optimal number of parameters to estimate, i.e. we expect that this number will decrease with $G B I C_{H A C}$ compared to $G B I C_{H C}$ due to the variance inflation factor.

2. Furthermore, if $G B I C_{H C, K L D} \neq G B I C_{H C}$, the model complexity term is strong and has an impact on the minimum of the criterion. This situation may arise for large samples. The same remark holds true for $G_{B B I} C_{H A C, K L D} \neq G_{B B I C_{H A C}}$.

3. We believe that in case of correlations, the most favourable case for the determination of the optimal numbers of parameters is obtained when $G B I C_{H C, K L D}=G B I C_{H A C, K L D}$ as it corresponds to a mitigation of $-\operatorname{tr}\left(\hat{\mathbf{H}}_{n}\right)+\log \left(\left|\hat{\mathbf{H}}_{n}\right|\right)$ by accounting for correlations, similarly to having $\mathbf{H}_{n}=\mathbf{I}$. In such a case, a balance between the model complexity and misspecification terms has been found. However, it does not mean that there is no correlation or that they can be neglected for further analysis (e.g. testing).

The previous comments are summarized in a flowchart in Figure 1.

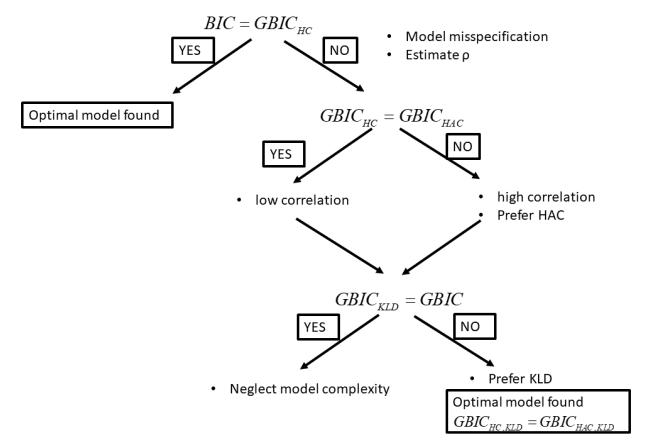

Fig. 1. Interpretation of the differences between the generalized BIC and the BIC by accounting for model complexity and misspecification

Thus, the disagreement of the criteria is in itself very important information. It provides useful indications about the goodness of fit of the estimation. The differences have to be further investigated, as didactically shown in the next section by means of dedicated examples. 


\section{Regression B-splines approximation}

In this section, we propose to apply the previous results to the particular case of determining the number of $\mathrm{CP}$ within the context of a regression B-spline curve. As mentioned in the introduction, the number of parameters for an approximation with B-splines functions is of main importance to ensure an optimal fitting to, e.g., the TLS observations, particularly when correlations and $\mathrm{HC}$ are present in the residuals of the adjustment.

For the sake of shortness, we will briefly explain how the approximation of scattered observations with Bsplines can be performed within the context of LS adjustment. Interested readers can refer to e.g. (Cox 1972, de Boor 1972, Piegl and Tiller 1997, Zhao et al. 2018) or Box and Jenkins (1976) for more details. In a second part, we will apply the derivation of Section 2 to determine the number of $\mathrm{CP}$ of the approximation to specific simulated B-spline curves. Correlations will be additionally artificially introduced in the simulated observations to study their impact on the different criteria.

\subsection{B-spline surface fitting}

In the following, we introduce the concept of B-spline surface approximation. Curve fitting with B-spline functions corresponds to a dimension reduction, i.e. the coordinates are $2 \mathrm{D}$ and the basis functions are computed in one direction only. The approximation of scattered observations with B-spline surfaces involve three main steps: (i) the parametrization of the data, (ii) the determination of the knot vector and, eventually, the degree of the basis functions $p$ and $q$, and (iii) the CP estimation. In the following, the two first steps are introduced briefly.

We define a 3D point of coordinates $[x(u, v), y(u, v), z(u, v)]$ depending on the two parameters $u$ and $v$ and lying on a B-spline surface by the tensor product of the B-spline basis functions $N_{i, p}$ and $N_{j, q}$ of degree $p$ and $q$, respectively:

$$
\mathbf{s}(u, v)=\sum_{i=0}^{n} \sum_{j=0}^{m} N_{i, p}(u) N_{j, q}(v) \mathbf{p}_{i, j}
$$

The points $\mathbf{p}_{i, j}$ are called CP. The B-spline basis functions are computed by a recursive formula (Cox 1972) and depends on the knot vector $\mathbf{U}=\left[\begin{array}{lll}u_{0} & \ldots & u_{k_{-} u}\end{array}\right], \mathbf{V}=$ $\left[\begin{array}{lll}v_{0} & \ldots & v_{k_{-} v}\end{array}\right]$ in the direction of the surface parameter $u$ and $v$, respectively, with $k \_u=n+p+1, k \_v=m+q+1$.
The knot vector is arranged in a non-decreasing order. Each of the basis functions is defined for the half-open interval $u \in\left[u_{i}, u_{i+1}\right)$ and $v \in\left[v_{j}, v_{j+1}\right)$, with $u_{i} \leq u_{i+1}, i=$ $0 \ldots n-1, \mathrm{v}_{j} \leq v_{j+1}, j=0 \ldots m-1$. The degrees of the basis functions are generally taken to 3 , which corresponds to cubic B-splines.

The parametrization of the surface consists of the determination of the location parameters $u$ and $v$ for each point of the point cloud. The chord length method mentioned in Piegl and Tiller (1997) gives satisfactory results for regular- and rectangular-shaped point cloud and will be used in this contribution. We assume that the point cloud represents a rectangular grid with $s$ rows and $t$ columns. For each row, the $k^{\text {th }}$ location parameters $u_{k}^{i}$ with $i=1, \ldots, s$ and $k=1, \ldots, t$ are calculated using the chord length method. To that aim, the Euclidean distance between adjacent points is calculated. The totalised Euclidean distance is then assigned to each point and normalized by a division through the totalised Euclidean distance assigned to the last point of the row. The average of the $s$ realisations of $u_{k}^{i}$ represents the parametrization in the $u$-direction. The computation in the $v$-direction proceeds in an analogous manner, see Alkhatib et al. (2018).

The determination of the knot vector is a nonlinear optimization problem (Gálvez et al. 2015), which can be solved using the knot placement technique, as described in Piegl and Tiller (1997). Alternatives would be based on, for example, the Monte Carlo method (Bureick, Alkhatib and Neumann 2016) but remain computationally demanding, particularly for surface fitting. Alternatively, the knot vector can be taken as equidistant.

The methodology to analyse the IC are not impacted by the choice of the knot vector, although the number found may vary (Alkhatib et al. 2018).

In the case of surface fitting, the $3 \mathrm{D}$ coordinates $\mathbf{x}_{C P}$ of the $\mathrm{CP}$ are estimated by $\hat{\mathbf{x}}_{C P}$, where the error term, i.e. the difference between the true and computed observations, is minimized in the LS sense. We call $\mathbf{y}$ the observation vector, sorted similarly to the deterministic design matrix $\mathbf{D}=\left[\begin{array}{ccc}\mathbf{D}_{\mathbf{x}} & \mathbf{0} & \mathbf{0} \\ \mathbf{0} & \mathbf{D}_{\mathbf{y}} & \mathbf{0} \\ \mathbf{0} & \mathbf{0} & \mathbf{D}_{\mathbf{z}}\end{array}\right]$ with $\mathbf{D}_{\mathbf{x}}=\mathbf{D}_{\mathbf{y}}=\mathbf{D}_{\mathbf{z}}=$ $\left[\begin{array}{ccc}N_{0, p}\left(u_{1}\right) N_{0, q}\left(v_{1}\right) & \ldots & N_{n, p}\left(u_{1}\right) N_{m, q}\left(v_{1}\right) \\ \ldots & \ldots & \\ N_{0, p}\left(u_{t}\right) N_{0, q}\left(v_{s}\right) & & N_{n, p}\left(u_{t}\right) N_{m, q}\left(v_{s}\right)\end{array}\right]$.

Thus, the global design matrix $\mathbf{D}$ is sorted component and not observation-wise, which is necessary to ensure the equivalence using the variance inflation factor. Assuming that the errors are normally distributed with $\mathbf{0}$ mean and have a constant variance, the LS adjustment equivalent to 
the MLE (Koch 1999) and the estimated parameter vector is given by

$$
\hat{\mathbf{x}}_{C P}=\left(\mathbf{D}^{T} \mathbf{D}\right)^{-1} \mathbf{D}^{T} \mathbf{y}
$$

We call $\hat{\mathbf{v}}=\mathbf{y}-\mathbf{D} \hat{\mathbf{x}}_{C P}$ the residuals of the LS adjustment. $\hat{\mathbf{x}}_{C P}$ is an unbiased estimator of the true parameters and not impacted by the choice of the stochastic model. Why is finding the optimal number of CP crucial?

The number of $\mathrm{CP}$ is linked with the length of the knot vector and usually fixed without prior knowledge, assuming that a high number will benefit the approximation. However, the avoidance of strong under- and overdetermination of the number of $\mathrm{CP}$, particularly for short samples, is crucial. This specific problematic of B-splines approximation is explained briefly in Figure 2, where a Gaussian noise vector was added to both components of a reference curve (green curve). This noisy curve was approximated using 5, 18 and $300 \mathrm{CP}$. Whereas a low number of CP (blue curve) leads to a strong departure from the true curve, a high number of CP (red curve) overfits the observations simulated, which is highlighted by the presence of oscillations in the fitted curve. Consequently, finding the optimal number of CP, here 18 , is essential. Please note that Figure 2 is a zoom in a more global curve, which explains the somehow strong difference between the blue curve and the three other ones.

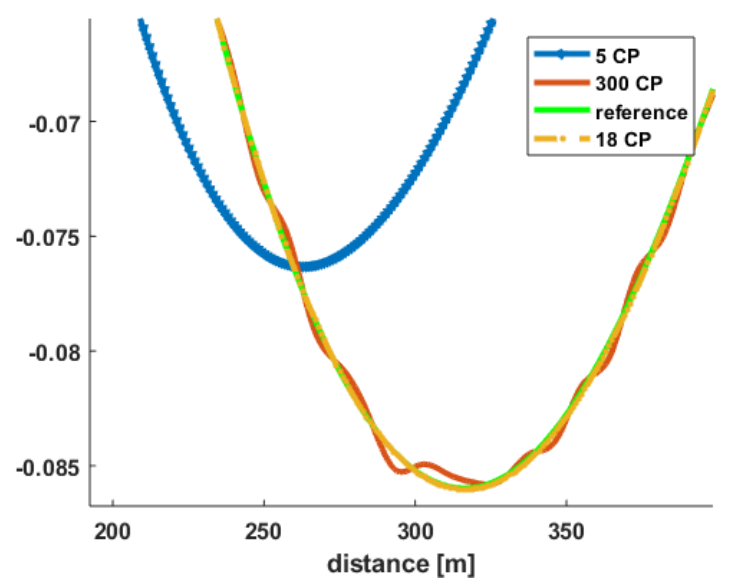

Fig. 2. Impact of an approximated determination of the number of $\mathrm{CP}$. The green reference curve represents a known B-spline curve to which white noise, having a variance of $1 \mathrm{~mm}^{2}$, was added. The blue curve corresponds to an underfitting of the simulated observations, whereas the red one is an overfitting, i.e. too many $\mathrm{CP}$, the noise being fitted instead of the curve.

\subsection{Case study}

In this section, we propose to analyse by means of simulations different case studies of B-spline curve approximations. The optimal number of $\mathrm{CP}$ will be determined with BIC or generalized BIC. The curves used in the following simulations are intentionally not generated by means of Bspline functions in order not to influence the BIC (Section 2). However, the condition $B I C=G B I C_{H C}$ ensures that the most optimal model is found, which we will call the "reference model".

Our focus is on a didactical interpretation of the results obtained with different criteria rather than a systematic analysis by means, for example, of Monte Carlo simulations.

\subsubsection{Simulated case study 1 : simple curve approximation}

The first simulated curve is depicted in Figure 3. It corresponds to a generation of 200 and 2000 equidistant points from the following function $y=0.3 \exp \left(-\frac{x}{200}\right) \cos \left(\frac{2 \pi x}{600}\right)$,.

In order to study the impact of the noise structure of the observations on the results given by the IC, two types of noise vectors were added to the parameter $y$ of the curve (Figure 3 right down):

- Case 1: Zero mean gaussian noise vector with a variance of $\sigma^{2}=1 \mathrm{~mm}^{2}$ and

- Case 2: Correlated AR(1) noise with a correlation coefficient of $\rho=0.8$ and a variance of $\sigma^{2}=1 \mathrm{~mm}^{2}$

The Matlab random generator number randn was used in both cases for the generation of a zero mean gaussian noise vector with a variance of $\sigma^{2}=1 \mathrm{~mm}^{2}$. For a concrete generation of the noise vector, interested readers should refer to Kermarrec and Schön (2016).

We approximate the curves using cubic B-splines and assume the homoscedasticity of the observations in the LS adjustment following Equation (10). Stochastic or functional model misspecifications are considered in the computation of the optimal number of CP by means of the derived quasi-likelihood criteria. These latter consider the potential heteroscedasticity as well as correlations of the residuals in their HC and HAC form, respectively. The results for the different samples sizes are presented in Table 1 . We varied the number of $\mathrm{CP}$ to estimate in a range of [3-100] by step of 1 and searched for the minimum of the IC. The knot vector was generated based on the current number of CP following (Piegl and Tiller 1997). For a given 

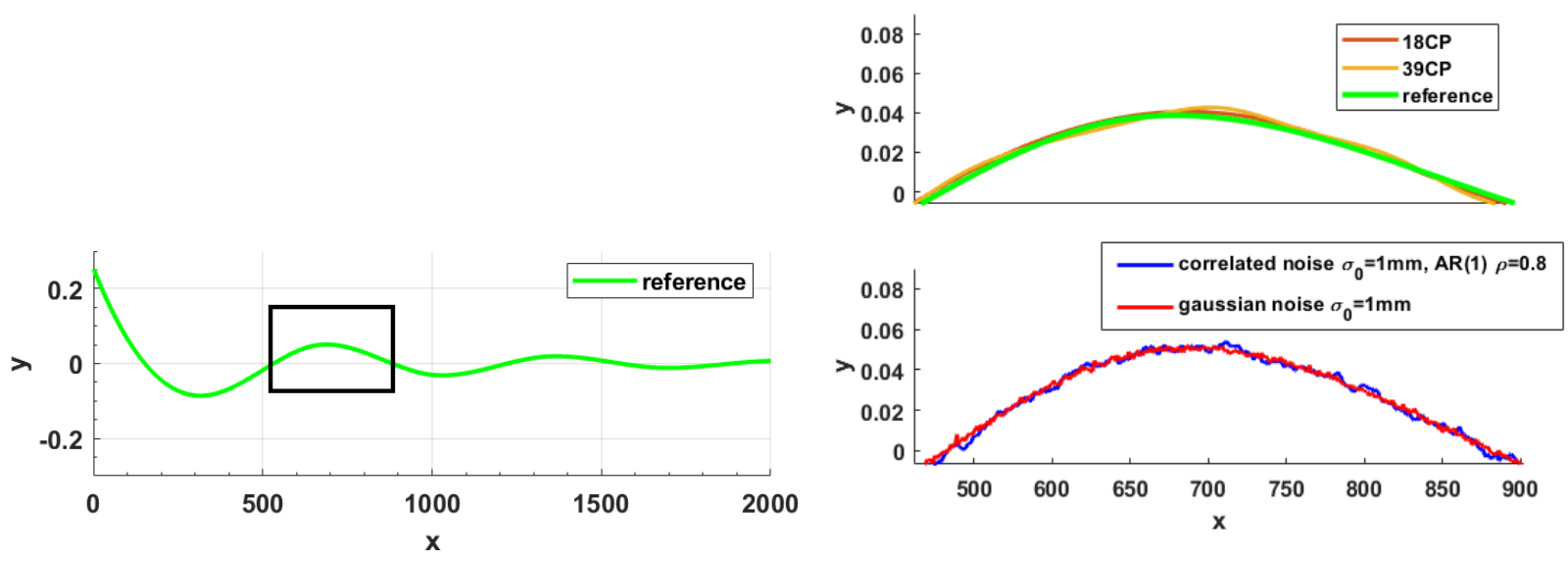

Fig. 3. Left: reference curve used for the simulations. Right down: zoom into the noisy curve corresponding to the black box. Correlated noise AR(1) with $\rho=0.8$ (blue) and Gaussian noise (red) were added to the reference curve. The noise variance factor was fixed to $\sigma^{2}=1 \mathrm{~mm}^{2}$. Right top: zoom into the fitted curves for different number of CP.

number of $\mathrm{CP}$, only the penalty terms (model misspecification and complexity) differ for the generalized IC (HC, HAC, and KLD versions).

Comments on the chosen AR(1) model

We justify the choice of the AR(1) function used to generate the correlated noise as a follow-up of the first empirical results obtained in Kauker and Schwieger (2017), where the correlation structure of least-squares residuals was approximated with this model. However, since model misspecifications also act as correlating the residuals, we do not expect the correlation structure of the residuals of the adjustment to follow exactly the chosen correlation function, i.e. we are not searching for $\rho_{\text {res }}=0.8$, with $\rho_{\text {res }}$ being the correlation coefficient of the residuals. Moreover, the AR(1) model is only an approximation of the correlation structure, which could be, e.g., modelled using a Matérn covariance function (Kermarrec and Schön 2017). Further detailed analysis are needed, which will be the topic of our next contributions.

\subsubsection{Results}

The BIC as well as the generalized IC were computed following the derivations of Section 2 for the four cases into consideration. Additionally, we investigated the minimum of the model misspecification terms $\log \left(\left|\hat{\mathbf{H}}_{H C}\right|\right)$ and $\log \left(\left|\hat{\mathbf{H}}_{H A C}\right|\right)$. Clearly, this term should not correspond to the minimum of the different IC but can be used to judge the strength of the misspecification by comparing its minimum with the reference or most optimal value. The num- ber of CP for which $\rho_{\text {res }}=0.8$ was reached is presented in the last line of Table 1.

Gaussian noise

From Table 1, first and third columns, we notice that the same optimal CP number of 18 is obtained for all IC. This result is coherent with the i.i.d. noise added to the observations, i.e. neither HC nor correlations impact the determination of the number of CP. No model misspecification affects the results of the fitting, which can be thus considered as optimal. Since $B I C=G B I C_{H C}$, we follow the results of Section 2 and conclude that the optimal number of $\mathrm{CP}$ is found. Consequently, $C P_{r e f}=18$ is here the reference value, for which the curve is approximated optimally using the chosen parametrization and knot vector determination method.

\section{Correlated noise}

- 2000 points

When the underlying noise is correlated, the optimal results given by the different IC differ. The number of CP determined by BIC (Table 1, fourth column) is higher than in the Gaussian case (Table 1, second column) and the global test failed with a confidence level of 0.05 , highlighting a strong model misspecification. Figure 3 (right top) highlights that the curve obtained from the BIC overfits the observations (orange curve), i.e. oscillations occur.

The optimal CP number obtained with BIC does not agree with the results obtained with generalized criteria, which is a strong evidence for the sub-optimality of the optimal number of CP given by the BIC. Following the flowchart presented in Figure 1, we found that: 
Table 1. Number of CP leading to the minimum value of each IC. 200 and 2000 points were generated and a Gaussian and a correlated noise (AR(1) with $\rho=0.8$ ) were added to the simulated curves.

\begin{tabular}{lllll}
\hline & $\begin{array}{l}\text { 200 points } \\
\text { Gaussian noise }\end{array}$ & $\begin{array}{l}\text { 200 points } \\
\text { Correlated } \\
\text { noise }\end{array}$ & $\begin{array}{l}\text { 2000 points } \\
\text { Gaussian noise }\end{array}$ & $\begin{array}{l}\text { 2000 points } \\
\text { Correlated } \\
\text { noise }\end{array}$ \\
\hline BIC & 18 & 49 & 18 & 186 \\
$G B I C_{H C}$ & 18 & 20 & 18 & 39 \\
$G B I C_{H A C}$ & 18 & 18 & 18 & 18 \\
$G B I C_{H C, K L D}$ & 18 & 20 & 18 & 18 \\
$G B I C_{H A C, K L D}$ & 18 & 18 & 18 & 18 \\
$\log \left(\left|\hat{\mathrm{H}}_{H C}\right|\right)$ & 18 & 16 & 18 & 57 \\
$\log \left(\left|\hat{\mathrm{H}}_{H A C}\right|\right)$ & 18 & 16 & 18 & 18 \\
$\rho_{\text {res }}=0.8$ & & 14 & & 17 \\
\hline
\end{tabular}

1. $\quad B I C \neq G B I C_{H C}:$ non-negligible model misspecification

2. $G B I C_{H C} \neq G B I C_{H A C}$ : presence of correlations in the residuals. The $G B I C_{H C}$ provides an optimal number of $39 \mathrm{CP}$, for which the global test failed. When correlations are additionally taken into consideration $\left(G B I C_{H A C}\right)$, the correction with $V F$ leads to an optimal number of parameters of $18 \mathrm{CP}$, for which the global test could be accepted. It corresponds to the reference $C P_{\text {ref }}$. Whereas $\log \left(\left|\hat{\mathbf{H}}_{H C}\right|\right)$ has a minimum for $57 \mathrm{CP}$, $\log \left(\left|\hat{\mathbf{H}}_{H A C}\right|\right)$ is minimal for a much lower value of 18 $\mathrm{CP}$, highlighting that the overdetermination could be optimally corrected with the $V F$, i.e. accounting for correlations decreases the number of $\mathrm{CP}$ to estimate. It has a comparable -and beneficial- effect as a filtering of the simulated observations, which corresponds to a lower number of CP necessary to fit the curve optimally.

3. $G_{B I C} C_{H C} \neq G B I C_{H C, K L D}$ : non-negligible model complexity term. A model with a smaller number of $\mathrm{CP}$ is preferably chosen.

4. $G B I C_{H A C, K L D}=G B I C_{H A C}$ : the value of the model complexity term $\operatorname{tr}\left(\hat{\mathbf{H}}_{n}\right)$ when correlations are accounted for is small, giving a strong indication that the optimal number of CP may have been found.

5. $G B I C_{H A C, K L D}=G B I C_{H C, K L D}$ : this result confirms the assumption of (iv)

Based on these considerations, we were able to find the optimal number of $\mathrm{CP}$ corresponding to $C P_{r e f}=18$. This value does not correspond to $\rho_{\text {res }}=0.8$ for the aforementioned reason. For the sake of completeness, we computed the generalized IC with a fully populated Toeplitz matrix corresponding to the AR(1) model. The same results were found as with the proposed simplification, which confirms empirically -and similarly to Kermarrec and Schön (2016) - that the equivalent diagonal matrix is a powerful alternative to fully populated VCM.

Figure 3 (right top) confirms visually that an optimal fitting is obtained. Please note that we intentionally chose not to compute the root mean square (RMS) of the difference between fitted and reference curves. Such statistical investigations are not the aim of this contribution, which intends to provide keys to interpret the BIC and generalized BIC. Moreover, we believe that this value does not give enough indication about the geometric goodness of the fitting. An oscillating curve due to overfitting is to our point of view not a correct approximation due to the lack of smoothness. To such a curve should be preferred one which may be far away from the original curve at some point but do not oscillate. We are aware that this point may be controversial.

- 200 points

In case of correlated observations with a small number of generated points, the same methodology can be applied to interpret the IC, which we summarize as follows:

1. $B I C \neq G B I C_{H C}$ : non-negligible model misspecification

2. $G B I C_{H C} \neq G B I C_{H A C}$ : presence of correlations in the residuals

3. $G_{B I C_{H C}}=G B I C_{H C, K L D}$ : negligible model complexity term, which we interpret as being due to the small amount of observations.

4. $G B I C_{H A C, K L D}=G B I C_{H A C}$ : see (iii)

5. $G B I C_{H A C, K L D} \neq G B I C_{H C, K L D}$ : the value given by $G B I C_{H A C, K L D}$ can be considered as optimal. It corresponds to $C P_{\text {ref }}=18$.

In all four cases we were able to identify the optimal number of $\mathrm{CP}$ to fit the given curve based on the interpretation of the differences between the IC. Only the combination of the criteria could provide a conclusion, i.e. it can never be 
claimed that one IC is better than the other one in all cases, which we illustrate with an additional case study.

\subsubsection{Simulated case study 2 : challenging geometry}

For the sake of completeness, a challenging curve with 400 points was additionally simulated (Figure 4, top). It has the equation $y=\frac{90}{1+\exp \left(x_{1 / 4^{-0.4}}\right)}$. The conclusions obtained by adding a Gaussian noise were similar to 3.3.1 and we found $C P_{\text {ref }}=36$. When an AR(1) noise with was superimposed to this reference curve (case 2), we found a maximum of $B I C$ for $179 \mathrm{CP}$, whereas $G B I C_{H C}$, $G B I C_{H C, K L D}$ and $G B I C_{H A C, K L D}$ agreed to an optimal solution of $36 \mathrm{CP}$. However, $G B I C_{H A C}$ led to an optimum for 41 $\mathrm{CP}$. Correlations are, thus, present in the residuals, which is confirmed by $V F=0.79$. Because $G B I C_{H A C, K L D}=$ $G B I C_{H C, K L D}$, we propose, following 3.3.1 to consider the number of 36 as optimal, which corresponds fortunately to $C P_{\text {ref. }}$. Interestingly, $G B I C_{H A C}>G B I C_{H C}$. This is an unexpected effect as the account for correlations should lead intuitively to a decrease of the optimal number of $\mathrm{CP}$. This result needs, thus, further investigations before concluding that $C P_{\text {ref }}=36$, as it implies a high and sensitive model misspecification term wrt. the model complexity and loglikelihood terms.

Figure 4 (down) highlights the different fitted curves obtained by varying the number of $\mathrm{CP}$. Within the distance 174-180 where the jump occurs, the number of CP given by $G B I C_{H A C, K L D}$ performed visually better than the one given by $G B I C_{H A C}$. The difference for the first jump of the reference curve (distance 130 from 140) is unfortunately in the opposite direction, so that $G B I C_{H A C}$ would be more optimal in this interval in terms of visual fitting and smoothness. Thus, both values could be considered as "good", see Gigerenzer and Marewski (2005), i.e. an improvement of the functional model by means of the parametrization or the knot vector, as well as variations of the degree of the basis functions should be investigated, combined with the results of the generalized BIC to find the optimal number of $\mathrm{CP}$ in presence of correlations.

\section{Real case study: surface approximation of TLS observations with B-splines}

The simulations have allowed to understand how the results of the different ICs can be interpreted to judge the
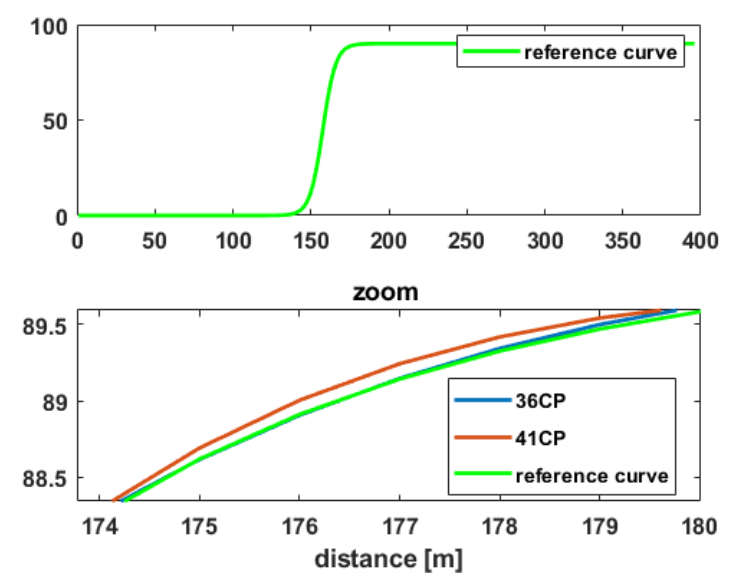

Fig. 4. (top) reference curve corresponding to a challenging case; (bottom) zoom for 2 approximations corresponding to 36 CP (blue), $41 \mathrm{CP}$ (red)

goodness of fit of noisy sampled curves approximated with B-splines functions. An interpretation of the results given by the IC was didactically provided in order to determine an optimal number of $\mathrm{CP}$ for the curve in case of correlated observations. We propose applying the previous findings to a case study using real TLS observations by extending the results obtained for curves to surface fitting of real TLS observations.

\subsection{Description of the dataset}

In this section, the optimal surface fitting of a 3D point clouds to a historic masonry arch bridge over the river Aller near Verden in Germany presented in Figure 5 is studied (Schacht et al. 2016). The aim of the underlying experiment was to investigate the detection of load-induced arch displacements by means of a laser scanner and other sensors. The TLS under consideration was a Zöller+Fröhlich Imager 5006, which captured 3D point clouds of the entire underside of the arch. After preparatory steps, including the removal of interfering objects such as prims, a data gap handling and a projection of the 3D point cloud on a regular grid, the B-spline approximation of the arch bridge could be performed using the raw measurements of the TLS, i.e. range and angles. Consequently, the transformation from polar to Cartesian coordinates induces mathematical correlations between the coordinates, so that the transformed observations are no longer i.i.d. TLS observations are moreover known to be heteroscedastic (Wujanz et al. 2018) with different variances for range and angles, as well as temporally correlated (Holst and Kuhlmann 2016, Holst et al. 2018). 


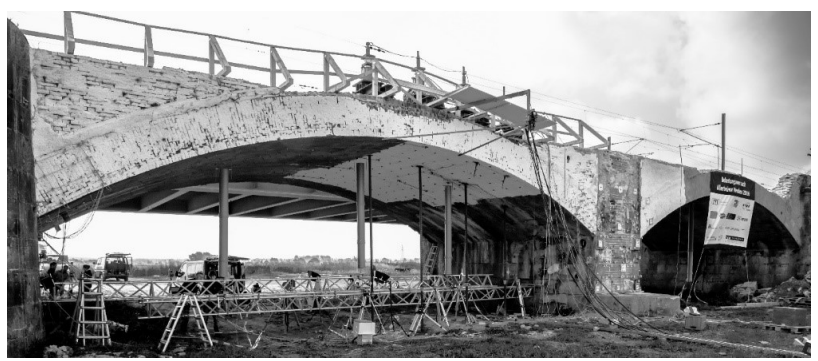

Fig. 5. Side view from the west of the historic masonry arch bridge under study. The challenge of data preprocessing was to eliminate unwanted objects from the point clouds (image from (Paffenholz, Huge and Stenz 2018))

Table 2. Optimal number of $\mathrm{CP}$ in the $u$ and $v$ directions for all criteria. Additionally, the minimum or inflexion point of the logdet term is given in the two last columns

\begin{tabular}{cccccccc}
\hline & BIC & $G B I C_{H C}$ & $G B I C_{H C, K L D}$ & $G B I C_{H A C}$ & $G B I C_{H A C, K L D}$ & $\log \left(\left|\hat{H}_{H C}\right|\right)$ & $\log \left(\left|\hat{H}_{H A C}\right|\right)$ \\
\hline$C P_{u}$ & $\mathbf{8}$ & $\mathbf{8}$ & $\mathbf{8}$ & $\mathbf{8}$ & $\mathbf{8}$ & 3 & $\mathbf{8}$ \\
$C P_{v}$ & $\mathbf{9}$ & 5 & 5 & 3 & 5 & 3 & 5 \\
\hline
\end{tabular}

The number of $\mathrm{CP}$ were varied in both directions $u$ and $v$, following the simulations. The solution of the IC is called $\left[C P_{u}, C P_{v}\right]$.

\subsection{Results for the IC analysis}

In this section, we analyse the results given by the IC without applying a test for significance (see Alkhatib et al. (2018)). Our approach has the advantage of being less computationally demanding than when, e.g., Vuong or Clarkes tests are used. Moreover, it allows, at the same time, a deeper understanding of the fitting, as highlighted in Section 3 by means of simulations.

To reduce the point cloud, a gridding was performed. A size of $80{ }^{\star} 120$ in direction $u$ and $v$ respectively was chosen for this case study, which corresponds to a $1.85 \mathrm{~cm}$ grid width in the $\mathrm{u}$-direction and $1.87 \mathrm{~cm}$ in the $\mathrm{v}$-direction. The coordinates were averaged inside a grid cell. Thus, this procedure reduces significantly the number of available observations. Acting as a filter, this method decreases the original correlations between raw observations in the gridded version of the point cloud.

Following the proposal of Section 2, we propose to approximate the correlation structure of the residuals of the LS adjustment with an AR(1) process. We found an inflation factor of 0.3 , highlighting a low level of correlations of the residuals. Table 2 presents the minima of the criterion as well as of the model misspecification terms obtained by varying the number of $\mathrm{CP}$.
Table 2 highlights that the traditional gives different optimal numbers of $\mathrm{CP}$ than the other criteria. As previously, we propose to summarize the most important conclusions as follows:

1. $\quad B I C \neq G B I C_{H C}$ : non-negligible model misspecification

2. $G B I C_{H C} \neq B G I C_{H A C}$ : presence of correlations in the residuals

3. $G B I C_{H C}=G B I C_{H C, K L D}:$ negligible additional model complexity term

4. $G B I C_{H A C, K L D}>G B I C_{H A C}$ : non-negligible additional model complexity term when accounting for correlations. $G B I C_{H A C, K L D}$ should be preferred as an underestimation of the number of $\mathrm{CP}$ can have severe consequence on the fitted surfaces wrt. a slight overestimation.

5. $\log \left(\left|\hat{\mathbf{H}}_{H A C}\right|\right)=[8,5]$ : because of the high number of points (more than 8000), we follow the results of the simulation and consider the minimum of the model misspecification term as corresponding to the optimal number of CP.

6. $G_{B I C_{H A C, K L D}}=G B I C_{H C, K L D}$ : confirms the assumption of (iv) and (v), i.e. $\left[C P_{u}, C P_{v}\right]=[8,5]$.

Our interpretation of the different IC leads to the choice of $\left[C P_{u}, C P_{v}\right]=[8,5]$. This is based on a combination of all the results found for the different criteria. In none of the case, the global test failed so that the BIC solution could be a possible alternative due to the low level of correlations and heteroscedasticity generated by the gridding of the PC. We do not claim that it is the best and unique solution. This one does not exist and, furthermore, depends on what we chose as being optimal, i.e. other possibilities than regression splines exist to approximate point clouds, which need to be further investigated within the context of engineering geodesy.

\section{Conclusion}

The control points define the shape of a B-spline curve or surface. Their position in space can be determined by minimizing the distance between the parametrized curve or surface and the observations in a LS sense. The total number of $\mathrm{CP}$ chosen affects the approximation strongly as well as the corresponding knot vector, which length has to be adapted correspondingly. Information criteria, such as BIC or AIC, provide a way to fix an optimal number of CP. However, their computation or derivation assumes i.i.d. errors. This is a strong limitation, particularly in the case of het- 
eroscedastic and/or correlated TLS range and angle measurements.

In this contribution, we used the concept of quasimaximum likelihood to adapt the penalty terms of BIC to account for these specific model misspecifications. Two additional terms were presented: (i) for model misspecification and (ii) for model complexity. A variance inflation factor was derived to resume the correlation structure of the errors into one term, thus, simplifying the fully populated VCM into weighted diagonal matrices. The corresponding generalized IC were applied to two detailed examples to provide a didactical way to interpret the results given from the different generalized IC and the BIC. It could be shown in which cases the results given by BIC can be considered as optimal and how correlations act on overestimating the optimal number of $\mathrm{CP}$ with the BIC. Thus, the necessity to account for correlations or $\mathrm{HC}$ in the computation within the context of the generalized IC was highlighted. More specifically, the introduction of a model misspecification term provides useful information about the goodness of fit, particularly in case of challenging geometries. Because none of the IC leads to the true solution which never exists in a real case - an understanding of the differences was identified as being crucial to avoid over- or underfitting and to be able to make an enlightened choice.

When applied to a real dataset, the generalized IC have been shown to lead to a reduction of the number of CP needed, compared with the traditional BIC. This highlights the importance of accounting for both heteroscedasticity and correlation of the residuals. Additionally, $\mathrm{HC}$ and correlations could be considered in the LS adjustment by means of the GLSE, using, for example, an adapted intensity variance model. The impact on the determination of the optimal number of CP remains to be further studied using the quasi-maximum likelihood IC, as well as the effect of data gaps or outliers. These points will be the purpose of further analysis based on the combined interpretation of the information criteria.

\section{References}

Aigner $M$ and Jüttler, B. Distance regression by Gauss-Newton-type methods and iteratively reweighted least-squares. Computing, 86 (2009), 73-87

Alkhatib H, Kargoll B, Bureick J and Paffenholz J-A. Statistical evaluation of the B-Splines approximation of 3D point clouds. Proceedings of FIG Congress 2018 Embracing our smart world where the continents connect: enhancing the geospatial maturity of societies, Istanbul, Turkey, May 6-11, 2018

Akaike $\mathrm{H}$. Information theory and an extension of the maximum likelihood principle. In: Petrov BN, Csaki F (eds) Proceedings of the 2nd International Symposium on Information Theory. Akademinai Kiado, Budapest, pp267-281 (1973)

Akaike H. A new look at the statistical model identification. IEEE Trans Autom Control, 19 (1974), 716-723

Atkinson A.C. A note on the generalized information criterion for choice of a model. Biometrika (1980), 67, 413-418

Box G.E.P., Jenkins G., Time series analysis, forecasting and control. Holden-Day, San Francisco, CA

Bureick J., Alkhatib H. and Neumann I. Robust spatial approximation of laser scanner points clouds by means of free-form curve approaches in deformation analysis. J Appl Geodesy, 10 (2016), 27-35

Burnham K.P. and Anderson D.A. Model selection and multimodel inference. Springer, New York (2002)

Bozdogan H. Model selection and Akaike's Information Criterion (AIC): The general theory and its analytical extensions. Psychometrika, 52 (1987), 345-370

Cherkassky V. and Mulier F. Learning from data. Wiley, Hoboken (2007)

Cox M.G. The numerical evaluation of B-splines. J Appl Math, 10 (1972), 134-149

de Boor C. On calculating with B-splines. J Approx Theory, 6 (1972), 50-62

Dziak J.J., Coffman D.L., Lanza S.T., Li R. Sensitivity and specificity of information criteria. Peer] Preprints. https://peerj.com/preprints/1103/ Accessed 18 December 2018

Foster D.P. and George E.I. The risk inflation criterion for multiple regression. Ann Statist 22 (1994), 1947-1975

Gálvez A., Iglesias A., Avila A., Otero C., Arias R. and Manchado C. Elitist clonal selection algorithm for optimal choice of free knots in B-spline data fitting. Appl Soft Comput 26 (2015), 90106

Gigerenzer G. and Marewski J. N. Surrogate science: The idol of a universal method for scientific inference. Journal of Management, 41, (2005), 421-440.

Harmening C. and Neuner H.B. Choosing the optimal number of Bspline control points (Part 1: Methodology and approximation of curves). J Appl Geodesy 10 (2016), 139-157

Harmening C. and Neuner H.B. (2017) Choosing the optimal number of B-spline control points (Part 2: Approximation of surfaces and applications). J Appl Geodesy 11 (2017), 43-52

Holst C. and Kuhlmann H. Challenges and Present Fields of Action at Laser Scanner Based Deformation Analysis. J Appl Geodesy 10 (2016),17-25

Kauker S. and Schwieger V. A synthetic covariance matrix for monitoring by terrestrial laser scanning. J Appl Geodesy 11 (2017) 77-87

Kermarrec G. and Schön S. (2016) Taking correlation into account with a diagonal covariance matrix. J Geodesy 90 (2016), 793805

Kermarrec G. and Schön S. (2017) Apriori fully populated covariance matrices in Least-squares adjustment - case study: GPS relative positioning. J Geodesy 91 (2017), 465-484

Kermarrec G, Alkhatib H. and Neumann I. On the sensitivity of the parameters of the intensity-based stochastic model for terrestrial laser scanner. Case study: B-spline approximation, Sensors, 18 (2018), 2964

Koch K.R. Parameter estimation and hypothesis testing in linear models, $2^{\text {nd }}$ ed, Springer, Berlin and New York (1999) 
Koch K.R. Fitting free-form surfaces to laserscan data by NURBS. Allgemeine Vermessungsnachrichten (AVN), 116 (2009), 134140

Kullback S. and Leibler R.A. On information and sufficiency. Ann Math Stat 22 (1951), 79-86

Liang K.Y. and Zeger S.L. Longitudinal data analysis using generalized linear models. Biometrika 73 (1986), 13-22

Lv J. and Liu J.S. Model selection principles in misspecified models. J.R. Statis. Soc. B, 76 (2014), 141-167

McCullagh P. Quasi-likelihood functions. Ann. Stat (1983), 11, 59-67

Paffenholz J.A., Huge J. and Stenz U. Integration von Lasertracking und Laserscanning zur optimalen Bestimmung von lastinduzierten Gewölbeverformungen. AVN 125 (2018), 75-89

Piegl L.A. and Tiller W. The NURBS book. Springer, Berlin, New York, (1997)

Rao C. and Toutenburg H. Linear models, least-squares and alternatives. Springer, New York, (1999)

Schacht G., Piehler J., Marx S. and Müller J.Z. Belastungsversuche an einer historischen Eisenbahn-Gewölbebrücke. Bautechnik 94 (2016), 125-130

Sclove S.L. Application of model-selection criteria to some problems in multivariate analysis. Psychometrika 52, (1987), 333343

Schwarz G. Estimating the dimension of a model. Ann Stat 6 (1978), 461-646

Tibshirani R. and Knight $K$. The covariance inflation criterion for adaptive model selection. J R Statist Soc B 61 (1999), 529-546

Wedderbrun R.W.M. Quasi-likelihood functions, generalized linear models and the Gauss-Newton method. Biometrika 61 (1974), 439-447

Wujanz D., Burger M., Mettenleiter M. and Neitzel F. An intensitybased stochastic model for terrestrial laser scanners. ISPRS 125 (2017), 146-155

Wujanz D., Burger M., Tschirschwitz F., Nietzschmann T., Neitzel F. and Kersten T.P. Determination of intensity-based stochastic models for terrestrial laser scanners utilising 3D-point clouds. Sensors 18 (2018), 2187

Xu P., Shi Y., Peng J., Liu J. and Shi C. Adjustment of geodetic measurements with mixed multiplicative and additive random errors. J Geod 87 (2013), 629-643 https://doi.org/10.1007/s00190-013-0635-2

Xu X., Kargoll B., Bureick J., Yang H., Alkhatib H. and Neumann I. TLS-based profile model analysis of major composite structures with robust B-spline method. Compos Struct 184 (2018), 814-820

Yanagihara H. Corrected version of AIC for selecting multivariate normal linear regression models in a general nonnormal case. J Multivar Anal 97 (2006), 1070-1089

Zhao X., Kargoll B., Omidalizarandi M., Xu X., Alkhatib H. Model selection for parametric surfaces approximating 3D point clouds for deformation analysis. Remote Sens 10 (2018), 634

Ziwot E. and Wang J. Modeling financial time series with S-plus. Springer Verlag, New York, (2003) 